\title{
voting method based on approval voting and arithmetic mean
}

\author{
Zoïnabo SAVADOGO ${ }^{1 *}$ and Blaise SOME ${ }^{2}$ \\ ${ }^{1}$ Laboratoire d'Analyse Numérique d'Informatique et de Biomathématiques (LANIBIO); Université Joseph KI-ZERBO (Burkina Faso). \\ ${ }^{2}$ Laboratoire d'Analyse Numérique d'Informatique et de Biomathématiques (LANIBIO); Université Joseph KI-ZERBO (Burkina Faso). \\ 1*serezenab@yahoo.fr \\ 2blaisesome@gmail.com
}

\begin{abstract}
Voting plays a vital role in any society. Indeed the votes involve decision making especially and the more in the decision of group. Thanks to the opinions expressed by a group of people, an opinion representing the preference of the group is determined. But most often some voting methods seem to distance the result from a vote of the general opinion. The study of voting methods is based on the theory of social choice. For several years, in the literature on the theory of social choice, many theorists have contributed trying to find a representative voting method.

It seems that there is no totally satisfactory way of voting.

Thus we have tried, through this article, to design a voting method based on approval voting and the arithmetic mean that leads to good compromise results.

In contrast to the other methods, the new method takes into account the choice of each voter and allows to obtain a result which represents the choice of the majority of the voters.
\end{abstract}

Keywords: Voting method-approval voting - arithmetic mean-Good compromise

\section{Introduction}

Literature is well supplied with many voting methods. In the group decision, several procedures have been proposed to determine, from individual preferences, a collective preference [7].

According to the same author, since the statement of the theorem of Arrow, several procedures have been proposed in the literature to escape this impossibility.

But until then it is difficult to say that this or that other method is totally satisfactory. According to [2], a voting system must, as a matter of priority, best reflect the preferences of voters.

Several criticisms are therefore made of these methods of voting by theoreticians of social choice and especially in relation to the controversial results they generate. Thus according to [9], there is an abundant literature on the aggregation of individual preferences into collective preference on the difficulties posed by this problem and on the strategic manipulation of social choice procedures as well.

Many writers have questioned the majority system, which seems to give sometimes surprising results.

Thus in [2], it appears that the ordinal approach of individual preferences, formerly dominant in social choice theory, is now disputed by some authors.

According to the same source, these authors reject classification voting systems and propose aggregation methods based on the evaluation principle.

According to [3] classification voting systems use ordinal preferences of voters and that this ordinal context does not make it possible to judge (or appreciate) the different options independently.

According to [6], the one or two-round majority voting system has a large number of widely known and proven defects.

The desire to find a voting system reflecting popular interest seems to be a great general concern.

Approval voting is a method with good properties according to the literature. It allows each voter to assess all candidates.

The elector is not restricted to voting for a single candidate. He may choose or, approve, several candidates, all or none if he wishes; but he can not vote several times for the same candidate. The winner is the candidate who has received the most approvals. This type of ballot, 
though simple, verifies properties very interesting and shows in many points superior to the majority vote [10].

Equally, approval voting can be defined as a procedure where each voter has the opportunity to express a cardinal preference in awarding a mark of one point to each candidate he or she supports and zero points to all others [2]. In addition to [4], approval voting is used during the selection of candidates for the position of Secretary-General of the United Nations Security Council and the Latvian parliamentary elections also draw inspiration from it.

In this work, we are inspired by the approval voting and the arithmetic mean, to contribute to the literature a satisfactory method.

\section{State of the art}

\subsection{Description of majority judgment}

This section is from [12]

Balinski and Laraki adopted, in their 2007 experience in the French presidential elections, the following common language:

\section{\{ Excellent, Very good, Good, Fairly, Fair, Insufficient, Refuse\}}

We call common language a set $\mathscr{L}=\left\{g_{1}, g_{2}, \ldots, g_{k}\right\}$ strictly ordered by" $>$ " such as $g_{1}>g_{2}>\ldots>g_{k}\left(g_{i} \geqslant g_{j}:=g_{i}>g_{j}\right.$ or $\left.g_{i}=g_{j}\right)$. Note that we can also have a common language be an infinite set such as the interval $[0,1]$ of real numbers with its natural order.

Note the possibility for a voter to assign the same assessment to more than two candidates. As such, a voter may award a $A$ candidate the VG score, a $B$ candidate the VG note and another $C$ candidate the note.G. In the context of arrow, we will say:

" A is at least as good as B ", " B is at least as good as A ", " $A$ is at least as good as C", " B is at least as good as $C$ ", " $A$ is preferred to $C$ ", " $B$ is preferred to $C$ ", " $A$ is indifferent to $B$ ".

A function $F$ is a ranking method if it associates to any profile a single rank [in the same language] for any candidate. So, $F: L^{m \times n} \longrightarrow L^{m}$

Where $m$ is the number of candidates, and $n$ the number of judges or voters.

Let $A_{i}$ be a candidate or competitor with grades $g_{i 1}, g_{i 2}, \ldots, g_{i n}$ where $g_{i 1} \geqslant g_{i 2} \geqslant \ldots g_{i n}$. Then the majority or majority grade $f^{m a j}\left[A_{i}\right]$ is by definition:

$$
f^{m a j}\left(A_{i}\right)= \begin{cases}f^{\frac{n+1}{2}}\left(g_{i 1}, g_{i 2}, \ldots, g_{i n}\right), & \text { if } n \text { is odd; } \\ f^{\frac{n+2}{2}}\left(g_{i 1}, g_{i 2}, \ldots, g_{i n}\right), & \text { if } n \text { is peer. }\end{cases}
$$

For example, if 5 judges award grades $4,8,7,9,5$ à $A_{i}$,

$$
f^{m a j}\left(A_{i}\right)=f^{3}(9,8,7,5,4)=7
$$

And if 8 judges award grades $9,7,3,6,5,4,5,8$ à $A_{i}$,

$$
f^{m a j}\left(A_{i}\right)=f^{5}(9,8,7,6,5,5,4,3)=5
$$

TIE-BREAKING [Balinski and Laraki, 2007]

When the majority grades of two candidates are different, the one with the highest rank is ranked before the other. The majority ranking $>_{m a j}$ between two candidates evaluated by the same jury is determined by a repeated application of the majority rank:

- If $f^{m a j}(A)>f^{m a j}(B)$ so $A>{ }_{m a j} B$

- If $f^{m a j}(A)=f^{m a j}(B)$ then a grade is rejected from the list of grades of each candidate and the procedure is repeated.

Balinski and Laraki [2010] give this example to illustrate their definition:

Suppose $A$ and $B$ are evaluated by a 7 voting jury:

\begin{tabular}{|l|l|l|l|l|l|l|l|}
\hline A & 85 & 73 & 78 & 90 & 69 & 70 & 73 \\
\hline B & 77 & 70 & 95 & 81 & 73 & 73 & 66 \\
\hline
\end{tabular}

The ordered profile is:

\begin{tabular}{|l|l|l|l|l|l|l|l|}
\hline A & 90 & 85 & 78 & $\underline{\mathbf{7 3}}$ & 73 & 70 & 69 \\
\hline B & 95 & 81 & 77 & $\underline{\mathbf{7 3}}$ & 73 & 70 & 66 \\
\hline
\end{tabular}

$f_{1}^{m a j}(A)=f_{1}^{m a j}(B)=73$. By definition, we reject 73 from both lists and we get:

\begin{tabular}{|l|l|l|l|l|l|}
\hline 90 & 85 & 78 & $\underline{\mathbf{7 3}}$ & 70 & 69 \\
\hline 95 & 81 & 77 & $\underline{\mathbf{7 3}}$ & 70 & 66 \\
\hline
\end{tabular}


$f_{2}^{m a j}(A)=f_{2}^{m a j}(B)=73$. By definition, we reject 73 from both lists and we get:

\begin{tabular}{|l|l|l|l|l|l|}
\hline A & 90 & 85 & $\underline{\mathbf{7 8}}$ & 70 & 69 \\
B & 95 & 81 & $\underline{\mathbf{7 7}}$ & 70 & 66 \\
\hline
\end{tabular}

$f_{3}^{m a j}(A)=78>f_{3}^{m a j}(B)=77$. Since then, $A>_{m a j} B$.

It is clear that the majority position always ranks one candidate before the other unless the judges give them the same rank.

In case there are several judges or voters [presidential elections for example], Balinski and Laraki present a way of dealing with tie-break.

The majority of a candidate with $f^{m a j}(A)=\alpha$ is a triplet $\left(p_{A}, \alpha^{*}, q_{A}\right)$ or $p$ is the number or percentage of the candidate's ranks that are greater than the majority rank, $q$ is the number or percentage of the candidate's ranks that are less than the majority rank, and $\alpha^{*}=\alpha^{+}$if $p>q$ et $\alpha^{*}=\alpha^{-}$if $p \leqslant q . \alpha^{*}$ is called the modified majority rank of the candidate.

By definition, $\alpha^{*}>\beta^{*}$ ssi $\alpha>\beta$ or $\left(\alpha=\beta\right.$ and $\alpha^{*}=\alpha^{+}$and $\left.\beta^{*}=\alpha^{-}\right)$. Balinski and Laraki use majority Gauge to define majority ranking $>_{m g}$.

Let $A$ and $B$ two candidates with respective majority gages $\left(p_{A}, \alpha_{A}^{*}, q_{A}\right)$ et $\left(p_{B}, \alpha_{B}^{*}, q_{B}\right)$.

So $A>_{m g} B$ or $\left(p_{A}, \alpha_{A}^{*}, q_{A}\right)>_{m g}\left(p_{B}, \alpha_{B}^{*}, q_{B}\right)$ ssi $\alpha^{*}>\beta^{*}$ or $\left(\alpha_{A}^{*}=\alpha_{A}^{*}=\alpha^{+}\right.$et $\left.p_{A}>p_{B}\right)$ or $\left(\alpha_{A}^{*}=\alpha_{A}^{*}=\alpha^{-}\right.$et $\left.p_{A}<p_{B}\right)$.

Manzoor Ahmed Zahid [2012] shows that ranking by majority can not decide between candidates in some cases. A theorem uttered by Balinski and Laraki (Theorem 14.1 in [Balinski and Laraki, 2010]) shows that:

$$
A>_{m g} B \Rightarrow A>_{m a j} B
$$

Ahmed Zahid then takes an example that illustrates a case where $A>_{m g} B$, but neither $B>_{m a j} A$ ni $B>_{m g} A$.

\begin{tabular}{|l|c|c|c|c|c|c|c|c|c|}
\hline Candidate & $p$ & Excellent & Very good & Good & Pretty good & Fair & A Reject & $q$ & Total \\
\hline A & $\underline{\mathbf{5}}$ & 2 & 3 & $\underline{\mathbf{3}}$ & 1 & 3 & 3 & 7 & 15 \\
\hline B & $\underline{\mathbf{6}}$ & 3 & 3 & $\underline{\mathbf{2}}$ & 0 & 2 & 5 & 7 & 15 \\
\hline
\end{tabular}

The majority $A$ is $\left(5, \operatorname{Good}^{-}, 7\right)$ And the one of $B$ is $\left(6, \operatorname{Good}^{-}, 7\right)$. As $q_{A}=q_{B}=7$, the majority rule does not make any decision and yet it is easy to verify that $A>_{m a j} B$.

\subsection{The mean-median compromise method (MCMM)}

This section comes from [12]

Drawing on the JM and BMC approaches, we are developing a method that can clearly and jointly address both theories and remove as many of the paradoxes presented above.

\subsubsection{Concepts and description of MCMM}

The method we present in this article is based on both the median and the mean. This is done by dividing the rank distribution for a candidate in $2^{k}$ intervals of the same magnitude. This division determines $2^{k}+1$ grades which are the points that bound the intervals. The function returns for evaluation of a candidate the Olympic average of $2^{k}+1$ grades retained.

Is $g_{1}, g_{2}, \ldots, g_{n}$ a sequence of $\mathrm{n}$ data such as $g_{1} \geqslant g_{2} \geqslant \cdots \geqslant g_{n}$. The Olympic average of this data is the number:

$$
\bar{g}_{o}=\frac{1}{n-2} \sum_{i=2}^{n-1} g_{i}
$$

The Olympic average of $n$ data is therefore the arithmetic mean of these data, put aside the two extreme values [largest and smallest].

Let $N$ be the set of $n$ judges, we call the amplitude of a division the real number:

$$
\alpha=\frac{n+1}{2^{k}} \text { with } k \geqslant 2
$$

$k$ is a pre-set integer called degree of division.

Let $A_{i}$ be a candidate or competitor with grades $g_{i 1}, g_{i 2}, \ldots, g_{i n}$ with $g_{i 1} \geqslant g_{i 2} \geqslant \cdots \geqslant g_{i n}$. A grade $g_{i j}$ is said inter-median if and only if $\exists m \in \mathbb{N}\left[\right.$ with $\left.1 \leqslant m \leqslant 2^{k}-1\right] \mid[\alpha . m]=j$ or $[\alpha . m]$ is the rounded value to the nearest number $\alpha . m$ and $\alpha$ the amplitude of the division for a division degree $k$ fixed.

We denote by $\mathscr{M}_{k}$ the set of non-redundant inter-median grades obtained with a division degree $k$.

$\mathscr{M}_{k}$ so defined is the set of data used in the calculation of the Olympic average of the points which are bounded [higher or lower] by the $2^{k}$ intervals obtained after division.

Let $A_{i}$ be a candidate or competitor with grades $g_{i 1}, g_{i 2}, \ldots, g_{i n}$ with $g_{i 1} \geqslant g_{i 2} \geqslant \cdots \geqslant g_{i n}$ and $\mathscr{M}_{k}=\left\{g_{i 1}^{\prime}, g_{i 2}^{\prime}, \ldots, g_{i j}^{\prime}\right\}$ all of his inter-median grades obtained with a degree of divisionk. Then the Medium Majority Compromise or Average Majority Rating or Average Majority Rank $f^{m m}\left(A_{i}\right)$ is by definition: 


$$
f^{m m}\left(A_{i}\right)=\frac{1}{j} \sum_{m=1}^{j} g_{\text {im }}^{\prime}
$$

For example, if 5 judges award grades $4,8,7,9,5$ à $A_{i}$. On se fixe $k=2, \alpha=\frac{5+1}{2^{2}}=1.5$

Ranks ranked in descending order give: $9,8,7,5,4$

$$
\mathscr{M}_{2}=\left\{f^{[1 \times 1.5]}, f^{[2 \times 1.5]}, f^{[3 \times 1.5]}\right\}=\left\{f^{2}, f^{3}, f^{5}\right\}=\{8,7,4\}
$$

So $f^{m m}\left(A_{i}\right)=\frac{8+7+4}{3}=\frac{19}{3}=6.33$.

And if 8 judges award grades $9,7,3,6,5,4,5,8$ à $A_{i}$. For $k=3, \alpha=\frac{8+1}{2^{3}}=1.125$

Ranks ranked in descending order give: $9,8,7,6,5,5,4,3$

$$
\begin{aligned}
\mathscr{M}_{3} & =\left\{f^{[1 \times 1.125]}, f^{[2 \times 1.125]}, f^{[3 \times 1.125]}, f^{[4 \times 1.125]}, f^{[5 \times 1.125]}, f^{[6 \times 1.125]}, f^{[7 \times 1.125]}\right\} \\
& =\left\{f^{[1.125]}, f^{[2.25]}, f^{[3.375]}, f^{[4.5]}, f^{[5.625]}, f^{[6.75]}, f^{[7.875]}\right\} \\
& =\left\{f^{1}, f^{2}, f^{3}, f^{5}, f^{6}, f^{7}, f^{8}\right\}=\{9,8,7,5,5,4,3\}
\end{aligned}
$$

So $f^{m m}\left(A_{i}\right)=\frac{9+8+7+5+5+4+3}{7}=\frac{41}{7}=5.8$

\subsubsection{TIE-BREAKING}

When the average majority grades of two candidates are different, the one with the highest average rank is ranked before the other. The majority ranking $>_{m m}$ between two candidates evaluated by the same jury is determined by a repeated application of the majority rank:

- We start with $k=2$

- If $f_{k}^{m m}(A)>f_{k}^{m m}(B)$ then $A>{ }_{m m} B$

- If $f_{k}^{m m}(A)=f_{k}^{m m}(B)$ then the procedure is repeated for $k+1$.

Let's take the following example to illustrate this definition:

Suppose that $A$ and $B$ are evaluated by a jury 7 voters:

\begin{tabular}{|l|l|l|l|l|l|l|l|}
\hline A & 85 & 73 & 78 & 90 & 69 & 70 & 71 \\
B & 77 & 72 & 95 & 83 & 73 & 73 & 66 \\
\hline
\end{tabular}

The ordered profile is:

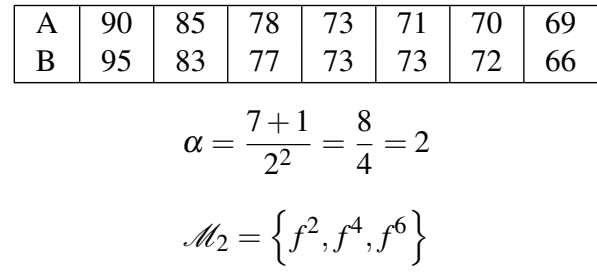

$\mathscr{M}_{2}[A]=85,73,70$ et $f_{2}^{m m}(A)=\frac{85+73+70}{3}=\frac{228}{3}=76$
$\mathscr{M}_{2}[B]=83,73,72$ et $f_{2}^{m m}(B)=\frac{85+73+72}{3}=\frac{228}{3}=76$

$f_{2}^{m m}(A)=f_{2}^{m m}(B)=76$. By definition, we repeat the procedure for $k=3$ and we obtain:

$$
\alpha=\frac{7+1}{2^{3}}=\frac{8}{8}
$$




$$
\begin{gathered}
\mathscr{M}_{3}=\left\{f^{1}, f^{2}, f^{3}, f^{4}, f^{2}, f^{6}, f^{7}\right\} \\
\mathscr{M}_{3}[A]=\{90,85,78,73,71,70,69\} \text { and } f_{3}^{m m}(A)=\frac{90+85+78+73+71+70+69}{7}=\frac{536}{7}=76.57 \\
\mathscr{M}_{3}[B]=\{95,83,77,73,73,72,66\} \text { and } f_{3}^{m m}(B)=\frac{95+83+77+73+73+72+66}{7}=\frac{539}{7}=77 \\
f_{3}^{m m}(B)=77>f_{3}^{m m}(A)=76.57 . \text { Then } f_{m m}(B)>f_{m m}(A) .
\end{gathered}
$$

In this example the average majority score gives exactly the same result as the average. This is due to the fact that the set of intermedian ranks of each competitor is equal to all of his ranks.

\section{Presentation of the new method}

The new method is also called the VMAVA (voting method based on approval voting and arithmetic mean) .

Consider a set $\mathrm{E}$ of $\mathrm{m}$ candidates for an election, with $m \geq 2$ and a set of voters with $s \geq 2$.

So the method is as follows:

Each of the voters uses elements of $\mathrm{P}(\mathrm{E})$ that are disjoint and whose meeting give $\mathrm{E}$, according to the following order: 1st choice, 2nd choice, 3 rd choice, 4th choice. He attributes to each element of each of his subsets respectively the note 4, 3,2, 1 .

The method consists first in considering the arithmetic mean of the points given by each voter to the candidates and then we use three sets as follows:

1. Gsup is made up of candidates who are above average

2. Gmoy is composed of candidates with exactly the average

3. Ginf is composed of candidates who have a score below average

Then we retain the candidates who are in the first class to know Gsup for each voter.

After we make the intersection of all Gsup, if we obtain a single candidate, it is the gagnant, if we get several candidates through this intersection, we reconsider the arithmetic average of their notes and we retain the one with the best average. In general suppose that we have $\mathrm{n}$ voters: if $\mathrm{n}$ is even, it is the candidate who belongs to at least $\frac{n}{2}+1$ Gsup who is the winner. If $\mathrm{n}$ is odd, we consider the integer part of $\frac{n}{2}$.

In case there are no candidates in the Gsup sets or if their intersection is empty, the procedure is repeated with the candidates in Gmoy. But these candidates are only moderately appreciated by the voters.

\begin{tabular}{|c|c|c|c|c|}
\hline $\mathrm{V} 1=$ & $\begin{array}{c}\text { 1st choice } \\
\left\{c_{2}, c_{4}\right\}\end{array}$ & $\begin{array}{c}\text { 2nd choice } \\
\left\{c_{1}\right\}\end{array}$ & $\begin{array}{c}\text { 3rd choice } \\
\left\{c_{3}\right\}\end{array}$ & 4th choice \\
\hline V2 & $\begin{array}{c}\text { 1st choice } \\
\left\{c_{1}\right\}\end{array}$ & $\begin{array}{c}\text { 2nd choice } \\
\left\{c_{3}\right\}\end{array}$ & $\begin{array}{c}\text { 3rd choice } \\
\left\{c_{4}\right\}\end{array}$ & $\begin{array}{c}\text { 4th choice } \\
\left\{c_{2}\right\}\end{array}$ \\
\hline $3=$ & $\begin{array}{c}\text { 1st choice } \\
\left\{c_{3}\right\}\end{array}$ & $\begin{array}{c}\text { 2nd choice } \\
\left\{c_{1}\right\}\end{array}$ & $\begin{array}{c}\text { 3rd choice } \\
\left\{c_{2}, c_{4}\right\}\end{array}$ & 4th choice \\
\hline $\mathrm{V} 4=$ & 1st choice & $\begin{array}{c}\text { 2nd choice } \\
\left\{c_{1}\right\}\end{array}$ & $\begin{array}{c}\text { 3rd choice } \\
\left\{c_{2}, c_{4}\right\}\end{array}$ & $\begin{array}{c}\text { 4th choice } \\
\left\{c_{3}\right\}\end{array}$ \\
\hline V5 & $\begin{array}{c}\text { 1st choice } \\
\left\{c_{1}\right\}\end{array}$ & $\begin{array}{c}\text { 2nd choice } \\
\left\{c_{3}\right\}\end{array}$ & 3rd choice & $\begin{array}{c}\text { 4th choice } \\
\left\{c_{2}, c_{4}\right\}\end{array}$ \\
\hline
\end{tabular}

If after all that there are no candidates in the Gmoy sets as well, it's better to review the elections if not using the Ginf sets, we will elect a candidate who is not well appreciated by the majority or who is not appreciated by anyone.

\subsection{Example of 4 candidates and 5 voters}

Using the correspondence giving the notes we have:

\begin{tabular}{|c|c|c|c|c|c|}
\hline & $c_{1}$ & $c_{2}$ & $c_{3}$ & $c_{4}$ & Moyenne \\
\hline V1 & 3 & 4 & 2 & 4 & 3.25 \\
\hline V2 & 4 & 1 & 3 & 2 & 2.5 \\
\hline V3 & 3 & 2 & 4 & 2 & 2.75 \\
\hline V4 & 3 & 2 & 1 & 2 & 2 \\
\hline V5 & 4 & 1 & 3 & 1 & 2.25 \\
\hline
\end{tabular}

For V1: Gsup $=\left\{c_{2}, c_{4}\right\} ;$ Gmoy $=\{\} ; \operatorname{Ginf}=\left\{c_{1}, c_{3}\right\}$

For V2: Gsup $=\left\{c_{1}, c_{3}\right\} ;$ Gmoy $=\{\} ; \operatorname{Ginf}=\left\{c_{2}, c_{4}\right\}$

For V3: Gsup $=\left\{c_{1}, c_{3}\right\} ; \operatorname{Gmoy}=\{\} ; \operatorname{Ginf}=\left\{c_{2}, c_{4}\right\}$

For V4: Gsup $=\left\{c_{1}\right\} ;$ Gmoy $=\left\{c_{2}, c_{4}\right\} ; \operatorname{Ginf}=\left\{c_{3}\right\}$

For V5: Gsup $=\left\{c_{1}, c_{3}\right\} ;$ Gmoy $=\{\} ; \operatorname{Ginf}=\left\{c_{2}, c_{4}\right\}$

The winner is $c_{1}$. 


\subsection{Example of 5 candidates and 4 voters}

\begin{tabular}{|c|c|c|c|c|}
\hline Notes & 4 & 3 & 2 & 1 \\
\hline Choices & 1st choice & 2nd choice & 3rd choice & 4th choice \\
\hline V1 & $\left\{c_{1}, c_{2}\right\}$ & & $\left\{c_{3}, c_{4}\right\}$ & $\left\{c_{5}\right\}$ \\
\hline V2 & & $\left\{c_{3}\right\}$ & $\left\{c_{1}, c_{2}\right\}$ & $\left\{c_{4}, c_{5}\right\}$ \\
\hline V3 & $\left\{c_{3}, c_{5}\right\}$ & $\left\{c_{1}\right\}$ & $\left\{c_{2}\right\}$ & $\left\{c_{4}\right\}$ \\
\hline V4 & $\left\{c_{1}\right\}$ & $\left\{c_{2}\right\}$ & $\left\{c_{3}\right\}$ & $\left\{c_{4}, c_{5}\right\}$ \\
\hline
\end{tabular}

Using the marks obtained by each candidate, we obtain the following table:

\begin{tabular}{|c|c|c|c|c|c|c|}
\hline & $c_{1}$ & $c_{2}$ & $c_{3}$ & $c_{4}$ & $c_{5}$ & Moyenne \\
\hline V1 & 4 & 4 & 2 & 2 & 1 & 2.6 \\
\hline V2 & 2 & 2 & 3 & 1 & 1 & 1.8 \\
\hline V3 & 3 & 2 & 4 & 1 & 4 & 2.8 \\
\hline V4 & 4 & 3 & 2 & 1 & 1 & 2.8 \\
\hline
\end{tabular}

The three sets per voter are:

For V1: Gsup $=\left\{c_{1}, c_{2}\right\}$; Gmoy $=\{\} ; \operatorname{Ginf}=\left\{c_{3}, c_{4}\right\}$

For V2: Gsup $=\left\{c_{1}, c_{2}, c_{3}\right\} ;$ Gmoy $=\{\} ; \operatorname{Ginf}=\left\{c_{4}, c_{5}\right\}$

For V3: Gsup $=\left\{c_{1}, c_{2}, c_{5}\right\} ;$ Gmoy $=\{\} ; \operatorname{Ginf}=\left\{c_{2}, c_{4}\right\}$

For V4: Gsup $=\left\{c_{2}, c_{3}\right\} ;$ Gmoy $=\{\} ; \operatorname{Ginf}=\left\{c_{3}, c_{4}, c_{5}\right\}$

Since we have 4 voters, it is the candidate who belongs to at least three Gsup at a time who is the winner so here, $c_{2}$.

\subsection{Example of 4 candidates and 4 voters}

\subsubsection{Example 1}

\begin{tabular}{|c|c|c|c|c|c|}
\hline & $c_{1}$ & $c_{2}$ & $c_{3}$ & $c_{4}$ & Moyenne \\
\hline V1 & 4 & 1 & 2 & 3 & 2.5 \\
\hline V2 & 1 & 4 & 2 & 3 & 2.5 \\
\hline V3 & 2 & 3 & 4 & 1 & 2.5 \\
\hline V4 & 3 & 3 & 1 & 3 & 2.5 \\
\hline
\end{tabular}

For V1: Gsup $=\left\{c_{1}, c_{4}\right\} ; \operatorname{Gmoy}=\{\} ; \operatorname{Ginf}=\left\{c_{2}, c_{3}\right\}$

For V2: Gsup $=\left\{c_{2}, c_{4}\right\} ; \operatorname{Gmoy}=\{\} ; \operatorname{Ginf}=\left\{c_{1}, c_{3}\right\}$

For V3: Gsup $=\left\{c_{2}, c_{3}\right\} ; \mathrm{Gmoy}=\{\} ; \mathrm{Ginf}=\left\{c_{1}, c_{4}\right\}$

For V4: Gsup $=\left\{c_{1}, c_{2}, c_{4}\right\} ; \operatorname{Gmoy}=\{\} ; \operatorname{Ginf}=\left\{c_{3}\right\}$

The winners are $c_{2}, c_{4}$ which are exequo, so we calculate the arithmetic average of the marks obtained by each of the two candidates, $c_{2}$ has $\frac{11}{4}$ and $c_{4}$ has $\frac{10}{4}$, so $c_{2}$ is the final winner.

\subsubsection{Example 2}

\begin{tabular}{|c|c|c|c|c|c|}
\hline & $c_{1}$ & $c_{2}$ & $c_{3}$ & $c_{4}$ & Moyenne \\
\hline V1 & 2 & 2 & 2 & 4 & 2.5 \\
\hline V2 & 1 & 4 & 2 & 3 & 2.5 \\
\hline V3 & 4 & 1 & 4 & 1 & 2.5 \\
\hline V4 & 3 & 3 & 1 & 3 & 2.5 \\
\hline
\end{tabular}

For V1: Gsup $=\left\{c_{4}\right\} ;$ Gmoy $=\{\} ; \operatorname{Ginf}=\left\{c_{1}, c_{2}, c_{3}\right\}$

For V2: Gsup $=\left\{c_{2}, c_{4}\right\} ;$ Gmoy $=\{\} ;$ Ginf $=\left\{c_{1}, c_{3}\right\}$

For V3: Gsup $=\left\{c_{1}, c_{3}\right\} ; \mathrm{Gmoy}=\{\} ; \mathrm{Ginf}=\left\{c_{2}, c_{4}\right\}$

For V4: Gsup $=\left\{c_{1}, c_{2}, c_{4}\right\} ;$ Gmoy $=\{\} ; \operatorname{Ginf}=\left\{c_{3}\right\}$

$c_{4}$ is the final winner.

\section{Comparison with the MCMM method}

Consider the following table: 


\begin{tabular}{|c|c|c|c|c|c|c|c|}
\hline & V1 & V2 & V3 & V4 & V5 & V6 & V7 \\
\hline$c_{1}$ & 2 & 1 & 2 & 1 & 3 & 3 & 4 \\
\hline$c_{2}$ & 4 & 1 & 1 & 4 & 3 & 3 & 1 \\
\hline$c_{3}$ & 2 & 1 & 2 & 1 & 4 & 3 & 3 \\
\hline$c_{4}$ & 3 & 3 & 3 & 3 & 3 & 3 & 3 \\
\hline$c_{5}$ & 4 & 4 & 1 & 3 & 3 & 4 & 1 \\
\hline Moyenne & 3 & 2 & 1.8 & 2.4 & 3.2 & 3.2 & 2.4 \\
\hline
\end{tabular}

For V1: Gsup $=\left\{c_{2}, c_{5}\right\} ; \mathrm{Gmoy}=\left\{c_{4}\right\} ; \operatorname{Ginf}=\left\{c_{1}, c_{3}\right\}$

For V2: Gsup $=\left\{c_{4}, c_{5}\right\} ;$ Gmoy $=\{\} ; \operatorname{Ginf}=\left\{c_{1}, c_{2}, c_{3}\right\}$

For V3: Gsup $=\left\{c_{1}, c_{3}, c_{4}\right\} ; \mathrm{Gmoy}=\{\} ; \operatorname{Ginf}=\left\{c_{2}, c_{5}\right\}$

For V4: Gsup $=\left\{c_{2}, c_{4}, c_{5}\right\} ; \mathrm{Gmoy}=\{\} ; \mathrm{Ginf}=\left\{c_{1}, c_{3}\right\}$

For V5: Gsup $=\left\{c_{3}\right\} ; \mathrm{Gmoy}=\{\} ; \mathrm{Ginf}=\left\{c_{1}, c_{2}, c_{4}, c_{5}\right\}$

For V6: Gsup $=\left\{c_{5}\right\} ;$ Gmoy $=\{\} ; \operatorname{Ginf}=\left\{c_{1}, c_{2}, c_{3}, c_{4}\right\}$

For V7: Gsup $=\left\{c_{1}, c_{3}, c_{4}\right\} ; \mathrm{Gmoy}=\{\} ; \operatorname{Ginf}=\left\{c_{2}, c_{5}\right\}$

Winners after the intersection of Gsup are: $c_{4}, c_{5}$ :

The arithmetic mean of $c_{4}$ is $\frac{21}{7}$ and that of $c_{5}$ is $\frac{20}{7}$.

The winner is $c_{4}$.

For $k=2, \alpha=\frac{7+1}{2^{2}}=2 ; M_{2}=\left\{f^{2}, f^{4}, f^{6}\right\}$ hence the following table:

\begin{tabular}{|c|c|c|c|}
\hline Candidates & grades & average MCMM & rank \\
\hline$c_{1}$ & 4332211 & $\frac{6}{3}=2$ & $4^{e}$ \\
\hline$c_{2}$ & 4433111 & $\frac{8}{3}$ & $2^{e}$ \\
\hline$c_{3}$ & 4332211 & $\frac{6}{3}=2$ & $4^{e}$ \\
\hline$c_{4}$ & 3333333 & $\frac{9}{3}=3$ & $1^{e r}$ \\
\hline$c_{5}$ & 4443311 & $\frac{8}{3}$ & $2^{e r}$ \\
\hline
\end{tabular}

For $k=3, \alpha=\frac{7+1}{2^{3}}=1$

\begin{tabular}{|c|c|c|c|}
\hline Candidates & grades & average MCMM & rank \\
\hline$c_{1}$ & 4332211 & $\frac{16}{7}$ & $4^{e}$ \\
\hline$c_{2}$ & 4433111 & $\frac{17}{7}$ & $3^{e}$ \\
\hline$c_{3}$ & 4332211 & $\frac{16}{7}$ & $4^{e}$ \\
\hline$c_{4}$ & 3333333 & $\frac{21}{7}$ & $1^{e r}$ \\
\hline$c_{5}$ & 4443311 & $\frac{20}{7}$ & $2^{\text {er }}$ \\
\hline
\end{tabular}

We find that both methods find the same result.

\section{Comparison with the majority judgment}

\begin{tabular}{|c|c|c|c|}
\hline Candidates & grades & majority judgment & rank \\
\hline$c_{1}$ & 4332211 & 2 & $4^{e}$ \\
\hline$c_{2}$ & 4433111 & 3 & $1^{e}$ \\
\hline$c_{3}$ & 4332211 & 2 & $4^{e}$ \\
\hline$c_{4}$ & 3333333 & 3 & $1^{e r}$ \\
\hline$c_{5}$ & 4443311 & 3 & $1^{e r}$ \\
\hline
\end{tabular}

According to the method, as there are exequo, we remove 3 from the list from where the following table:

\begin{tabular}{|c|c|c|c|}
\hline Candidates & grades & majority judgment & rank \\
\hline$c_{2}$ & 443111 & 3 & $2^{e}$ \\
\hline$c_{4}$ & 333333 & 3 & $2^{e}$ \\
\hline$c_{5}$ & 444311 & 4 & $1^{e r}$ \\
\hline
\end{tabular}

We find that candidate 4 is the winner with the VMAVA method and the MCMM method. While the majority judgment finds that candidate 5 is the winner.

One of the advantages of VMAVA is that it allows to elect a candidate who at least appreciated by the majority. It allows donation to reduce the disputes encountered after the votes.

\section{Discussion and Perspectives}

The aggregation of individual preferences into a collective preference is conceived by means of an aggregation function, which are generally mathematical formulas or functions of social choice. This formula makes it possible to classify the candidates to an election through the appreciation of the voters on these last ones. The determination of a social choice function with good properties makes it possible to transform the individual choice into a choice representing the general interest which is the desire sought. The VMAVA is a method with good 
properties because it does not compensate for strong criteria by weak ones like the arithmetic mean which is widely used in many voting systems. It favors candidates who do not have very weak scores and who are not very dispersed. Voting by approval being well appreciated in the literature because of its interesting properties, and allowed the development of the VMAVA method which also fulfills good properties. We have therefore brought to the literature a good voting system in the sense that its use does not give controversial results. Certainly it could engender some limits as any function of social choice according to [1], the choice of a voting method shapes the democracy in which we live. A "good" voting system, fulfilling all the desirable properties, does not exist: one has to be satisfied with a satisfactory vote [2].

In addition to [4], it appears that the experimental results confirm that, for given preferences, changing the voting system is likely to change the outcome of the election. Similarly in [5], several recent studies also highlight the fact that Aggregate results differ from one voting system to another.

\section{References}

[1] Antoinette BAUJARD et al: vote par approbation, vote par note, une expérimentation lors de l'élection présidentielle du 22 avril 2012 , revue économique 2013/2-vol.64 pages 345 à 356

[2] Antoinette BAUJARD, Herrade IGERSHEIM: Expérimentation du vote par note et du vote par approbation de l'élection présidentielle française du 22 avril 2007, Rapport final, Antoinette BAUJARD, CREM, Université de Caen-Basse-Normandie; Herrade IGERSHEIM, CNRS et CEPERC, Université de Provence-Aix-Marseille I, Rapport public du Centre dAnalyse Stratégique. 2007. ¡halshs-00337290i 2007

[3] Hatem SMAOUI, Dominique LEPELLEY: Le système de vote à trois niveaux: Etude d'un nouveau mode de scrutin, Dalloz| «Revue d'économie politique $\gg, 2013 \mid 6$ vol.123| pages 827 à 850 ISSN 0373-2630

[4] Herrade IGERSHEIM, Antoinette BAUJARD, Jean-François LASLIER: La question du vote. Expérimentations en laboratoire et In Situ., Working paper GATE 2016-33. 2016.; halshs-01402275;

[5] Herrade IGERSHEIM, Antoinette BAJARD, Jean-François LASLIER: L'actualité économique,Revue d'analyse économique, vol.92, no1-2, mars-juin 2016

[6] Jorge Gonzalez SUITT, Axel GUYON, Thibault HENNION, Rida LARAKI, Xavier STARKLOFF, Sophie THIBAULT, Benjamin FAVREAU: Vers un système de vote plus juste?, cahier no 2014-20, septembre 2014.

[7] Khaled JABEUR, Jean-Marc MARTEL: Une méthode de choix collectif à partir de systèmes relationnels de préférences (S.R.P.) , ISBN-2-89524-202-X, 04-2004.

[8] Michel BALINSKI,Rida LARAKI, Jugement majoritaire vs. vote majoritaire,cahier no 2012-37, Département d'économétrie, Route de Saclay, 91128 PALAISEAU CEDEX (33) 169333033 .

[9] Michel TRUCHON: Choix social et comités de sélection: le cas du patinage artistique, Université Laval CIRANO,CIRPEE Novembre 2002 , ISSN 1701-9990, 2002RB-02

[10] Patrick BLANCHENAY: Parodoxes de vote et modes de scrutin en France, Ecole des hautes études commerciales Majeure économie, Mai 2004, sous la direction de Hervé CRES

[11] Roderick J. WOOD: Rapport de la commission du droit du Canada sur la réforme électorale, Mémoire, Université de l'Alberta , 29 Septembre 2016

[12] Ruffin-Benoît M. NGOIE, Zoinabo SAVADOGO, Berthold E.-L.ULUNGU: Median and average as tools for measuring, electing and Ranking: New propects, Fundamental Journal of Mathematics and Mathematical Sciences, vol.1, Issue 1, pages 9-30, 2014 